\title{
Grounding Resistance of a Hemispheric Electrode Located on the Top of a Finite-Height, Cone-Shaped Mountain
}

\author{
Antonio Sunjerga, Marcos Rubinstein, Fellow, IEEE, Dragan Poljak, Senior Member, IEEE, Hamidreza \\ Karami and Farhad Rachidi, Fellow, IEEE
}

\begin{abstract}
In this study, we present an analytical solution for the resistance of a hemispheric grounding electrode located on the top of a mountain. The mountain is modeled as a truncated cone with a finite height. Recently, a closed-form solution for the grounding resistance was derived first for a hemispheric electrode on top of a cone, and later for a more realistic case of a truncated cone with a flat region at its top. The height of the cone was considered infinite in those studies. Here, we extend these studies for the case of a truncated cone with finite height. The analytical solution is compared with numerical simulations and the results agree reasonably well.
\end{abstract}

Index Terms - Lightning, grounding resistance, hilly terrain, elevated terrain, grounding electrode, finite height

\section{INTRODUCTION}

$\mathrm{M}$ ountain tops are ideal locations for placing either telecommunication towers to obtain line of sight, or wind turbines to maximize the generated power. These locations have a higher risk of being struck by lightning due to the geographical elevation and the height of the tower itself [1-3]. Furthermore, tall structures located on mountaintops can also initiate upward flashes [4]. Furthermore, these areas being often characterized by low soil conductivities, lightning protection of these structures is a challenging task $[5,6]$.

The effectiveness of a grounding system depends mostly on its geometry and its surrounding soil properties, such as the electrical properties of the ground, their frequency dependence and the soil stratification (e.g., [7,8 and 9]), as well as soil ionization [e.g., 10].

It has recently been shown that the grounding resistance can be significantly increased in the case of a non-flat terrain that effectively reduces the conductive volume for the injected current [11]. A similar degree of increase was obtained for both, hemispheric grounding electrodes [11] and vertical rods [12], suggesting that the increase in the grounding resistance is mostly governed by the soil geometry and not by the geometry of grounding electrode. Remote grounding can be one effective way of reducing the grounding resistance of structures located on mountaintops [13].

Analytical solutions for the calculation of the grounding resistance of electrodes buried in the soil are available in the case of a flat terrain (e.g. [14-17]). Recently, analytical solutions for the low-frequency response of a hemispheric grounding electrode buried on the top of a cone-shaped mountain characterized by its apex angle were derived $[11,18]$.
In these studies, the height of the mountain was assumed to be infinite.

In this letter, we present an analytical solution for the grounding resistance of a hemispheric grounding electrode located on the top of a truncated cone, characterized by a finite height.

\section{ANALYTiCAL SOLUTIONS}

Fig. 1 presents three different simplified geometries representing a hemispheric grounding electrode located on the top of a mountain. The models shown in Fig. 1-a and Fig. 1-b have been considered in [11]. In these models, the height of the cone-shaped mountain was assumed to be infinite. In this study, we will relax this assumption and consider the geometry shown in Fig. 1-c, in which the finite height of the mountain is taken into account.

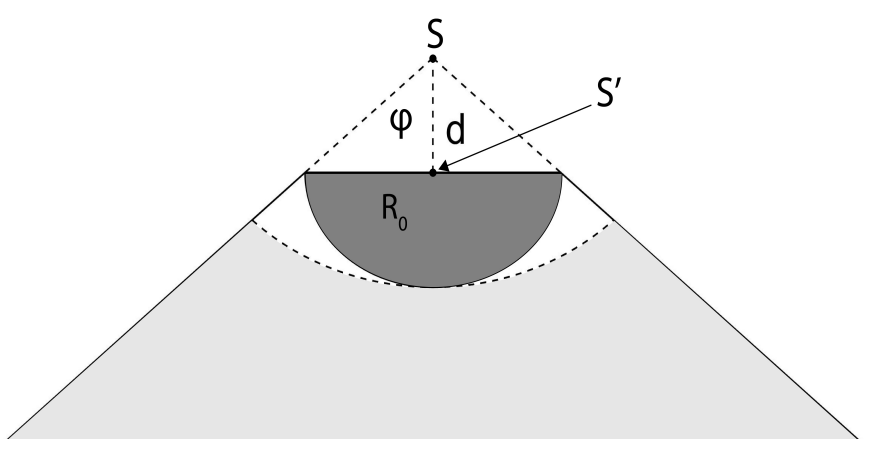

(a)

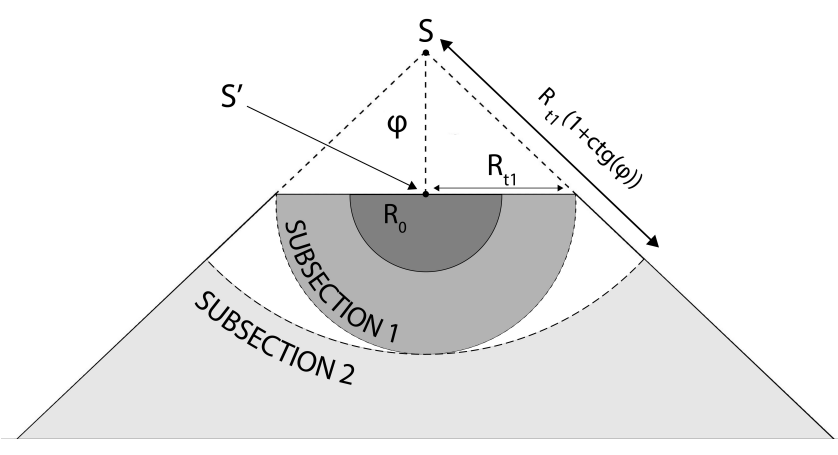

(b) 


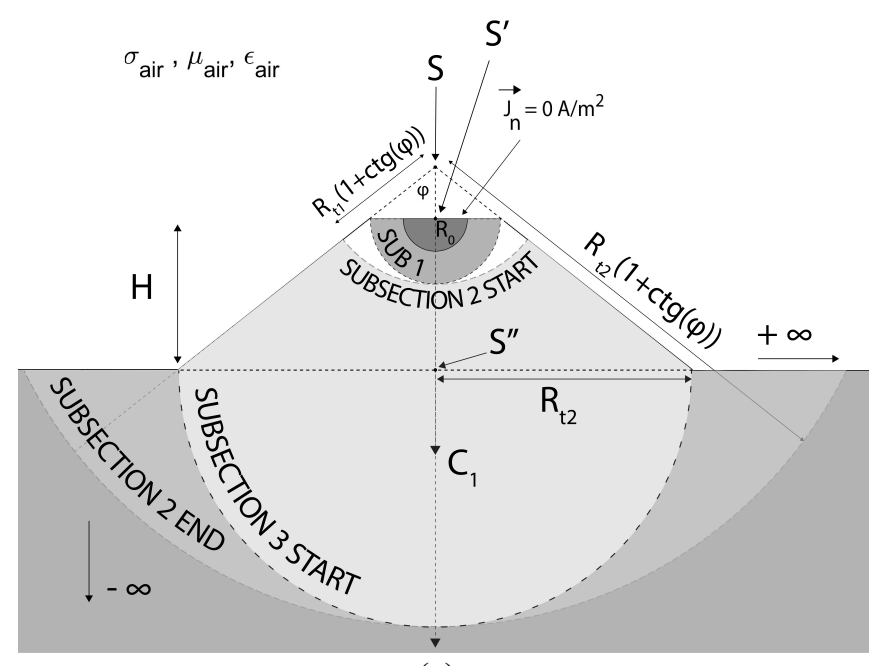

(c)

Fig. 1. Hemispheric grounding electrode in three different geometries of the soil. (a) Electrode buried on the top of a truncated cone-shaped ground. The top radius of the cone is assumed to be equal to the radius of the electrode (b) Same as in (a) but the top radius of the cone is bigger than the radius of hemispheric electrode (c) Same as in (b) but considering a cone-shaped mountain with a finite height.

The analytical solution for the grounding resistance for the simplified model shown on Fig. 1-a is given in [11]:

$$
R_{a}=\frac{1}{2 \pi \sigma R_{0}(1-\cos (\varphi))(1+\operatorname{ctg}(\varphi))}
$$

where $\varphi$ is the apex angle of the cone, $\sigma$ is the soil conductivity, and $R_{0}$ is the radius of hemispheric electrode.

In [18], the solution for the model shown in Fig. 1-b was obtained, splitting the soil into two subsections and summing the respective potentials. The two subsections are illustrated in Fig. 1-b The potential of the first subsection is governed by equations related to a flat ground, while the potential for the second one is governed by the same set of equations corresponding to the geometry of Fig. 1-a [11]. The derived expression for the grounding resistance is [18]:

$$
R_{b}=\left(\frac{1}{R_{0}}-\frac{1}{R_{t 1}}+\frac{1}{R_{t 1}(1-\cos (\varphi))(1+\operatorname{ctg}(\varphi))}\right) \frac{1}{2 \pi \sigma}
$$

in which $R_{t 1}$ is the distance from the center of the hemisphere $\mathrm{S}$ ' to the edge of the cone (see Fig. 1-b).

Using a similar approach as the one used in [18], one can divide the geometry in Fig. 1-c into three subsections. Each subsection in the figure is an annulus sector formed by the space between two arcs of the same circle as follows: Subsection 1, labeled SUB1 in Fig. 1-c, is the annulus sector centered at S' and bounded by the arcs with radii $R_{0}$ and $R_{t 1}$ (the latter not labeled explicitly in the figure). Subsection 2 is the annulus sector centered at $\mathrm{S}$ and bounded by the circular arcs with radii $R_{t 1}(1-\cos (\varphi))$ and $R_{t 2}(1-\cos (\varphi))$. Finally, subsection 3 is the annulus sector centered at $\mathrm{S}$ " and bounded by the arcs of radii $R_{t 2}$ and $\infty$.

As can be seen from Fig. 1-c, the first and third subsections are governed by the equations associated with a flat ground. The second subsection is governed by the equations associated with the geometry presented in Fig. 1-a and given in [11] with the origin of the coordinate system at the tip of the cone. Note that the region between subsections one and two shown in white in Fig. 1-c is not part of any of the considered subsections and it is the result of the adopted approximation. On the other hand, the region delimited by the start of subsection 3 and the end of subsection 2 belongs to both, subsections 2 and 3. In the following analysis, the potential will be calculated along the curve $C_{1}$ (shown in Fig. 1-c.) in order to avoid these undefined regions.

The electrode potential with respect to the remote earth can be obtained as:

$$
V_{\infty} \approx \int_{r^{\prime}=R_{0}}^{r^{\prime}=\infty} \vec{E} \cdot \overrightarrow{d r^{\prime}}
$$

This voltage can be obtained by integrating the electric field along the curve $C_{1}$ and splitting the domain into the three subsections as shown in Fig. 1-c.

First, the voltage drop along $C_{1}$ in the first subsection can be obtained using the flat ground voltage expression with the origin of the coordinate system being the center of the hemisphere electrode marked with S' (Fig 1-c):

$$
V_{1} \approx \int_{r^{\prime}=R_{0}}^{r \prime=R_{t 1}} \vec{E} \cdot \overrightarrow{d r^{\prime}}=\left(\frac{1}{R_{0}}-\frac{1}{R_{t 1}}\right) \frac{I}{2 \pi \sigma}
$$

where $I$ is the injected current.

The voltage drop along $C_{1}$ in the second subsection can be obtained using the expression derived in [11], considering the origin of the coordinate system at the tip of the cone marked with S (Fig 1-c):

$$
\begin{gathered}
V_{2} \approx \int_{r=R_{t 1}(1+\operatorname{ctg}(\varphi))}^{r=R_{t 2}(1+\operatorname{ctg}(\varphi))} \vec{E} \cdot \overrightarrow{d r} \\
=\left(\frac{1}{R_{t 1}(1-\cos (\varphi))(1+\operatorname{ctg}(\varphi))}\right. \\
\left.-\frac{1}{R_{t 2}(1-\cos (\varphi))(1+\operatorname{ctg}(\varphi))}\right) \frac{I}{2 \pi \sigma}
\end{gathered}
$$

Finally, the voltage drop along $C_{1}$ in the third subsection can be approximated using the flat earth expression considering the origin of the coordinate system shown with S" (Fig 1-c):

$$
V_{3} \approx \int_{r^{\prime \prime}=R_{t 2}}^{r \prime \prime=\infty} \vec{E} \cdot \overrightarrow{d r^{\prime \prime}}=\frac{I}{2 \pi \sigma R_{t 2}}
$$

The total electrode potential is equal to the sum of these three terms:

$$
\begin{aligned}
V_{\infty}= & V_{1}+V_{2}+V_{3} \\
= & \left(\frac{1}{R_{0}}-\frac{1}{R_{t 1}}+\frac{1}{R_{t 1}(1-\cos (\varphi))(1+\operatorname{ctg}(\varphi))}\right. \\
& \left.-\frac{1}{R_{t 2}(1-\cos (\varphi))(1+\operatorname{ctg}(\varphi))}+\frac{1}{R_{t 2}}\right) \frac{I}{2 \pi \sigma}
\end{aligned}
$$


in which $R_{t 2}$ is:

$$
R_{t 2}=\tan (\varphi) H+R_{t 1}
$$

Dividing (7) by the current, one can obtain the expression for the grounding resistance as:

$$
\begin{aligned}
R_{c}= & \left(\frac{1}{R_{0}}-\frac{1}{R_{t 1}}+\frac{1}{R_{t 1}(1-\cos (\varphi))(1+\operatorname{ctg}(\varphi))}\right. \\
& \left.-\frac{1}{R_{t 2}(1-\cos (\varphi))(1+\operatorname{ctg}(\varphi))}+\frac{1}{R_{t 2}}\right) \frac{1}{2 \pi \sigma}
\end{aligned}
$$

It can readily be shown that when the mountain height tends to infinity, (8) tends to infinity as well, so that (9) reduces to (2). Furthermore, it can easily be shown that imposing $\mathrm{R}_{\mathrm{t}}=\mathrm{R}_{0}$, (2) will be reduced to (1).

\section{VALIDATION With Numerical SimUlations}

In this section, we present a comparison between the derived analytical solution (9) and numerical simulations obtained using the commercial software COMSOL [19]. More details about simulations can be found in [11]. Table 1 presents the numerical results considering different values for the height and apex angle for the studied geometry (Fig. 1-c). It can be seen that the assumption of an infinite mountain height results in an overestimation of the grounding resistance. Note that we only considered a single conductivity of $0.001 \mathrm{~S} / \mathrm{m}$ in our calculations. The grounding resistance has a linear dependency with the soil conductivity, as can be readily seen in the analytical equation (9) and confirmed by numerical evaluation.

As the apex angle increases toward the limit of $90^{\circ}$ and the height to the limit of zero, the grounding resistance will tend to the value corresponding to a flat ground:

$$
R_{\text {flat }}=\frac{1}{2 \pi \sigma R_{0}}
$$

which is equal to $31.8 \Omega$ for the observed case.

The relative errors of equation (9) are presented in Table 2.

\begin{tabular}{|c|c|c|c|c|c|}
\hline \multirow{2}{*}{$\begin{array}{l}\text { Apex } \\
\text { angle } \\
(\varphi)\end{array}$} & \multicolumn{4}{|c|}{ Resistance $(\Omega)$} & \multirow[b]{2}{*}{$H=\infty$} \\
\hline & $\mathrm{H}=10 \mathrm{~m}$ & $\mathrm{H}=25 \mathrm{~m}$ & $\begin{array}{c}\mathrm{H}=100 \\
\mathrm{~m}\end{array}$ & $\begin{array}{c}\mathrm{H}=250 \\
\mathrm{~m}\end{array}$ & \\
\hline 45 & 32 & 35.7 & 39.2 & 41.3 & 46.2 \\
\hline 30 & 36.3 & 44.4 & 54 & 57.5 & 62.9 \\
\hline 15 & 42.2 & 60.3 & 92.2 & 105.8 & 121 \\
\hline 10 & 44.7 & 69.1 & 121.2 & 148.6 & 188.7 \\
\hline
\end{tabular}
We can observe that the results obtained using the analytical solutions agree reasonably well with the reference numerical results.

Fig. 2 presents a plot of the increase of the grounding resistance as a function of the cone height and apex angle compared to the case of a flat ground. The grounding resistance was obtained analytically using (5). As the value of the apex angle increases, the results converge to those corresponding to the case of a flat ground. In a similar way, decreasing the height, the results converge to those of a flat ground. For the case of an apex angle of $30^{\circ}$ and a height of about $100 \mathrm{~m}$, the increase of the grounding resistance is almost a factor of two.

\begin{tabular}{lccccc}
\hline TABLE 2. Grounding resistance relative error of eq. (9) for $\sigma=0.001 \mathrm{~S} / \mathrm{m}$, \\
$\mathrm{R}_{0}=5 \mathrm{~m}, \mathrm{R}_{\mathrm{t} 1}=10 \mathrm{~m}$, and $\mathrm{R}_{\mathrm{flat}}=31.8 \Omega$ \\
\hline \multicolumn{5}{c}{ Resistance $(\Omega)$} \\
$\begin{array}{l}\text { Apex } \\
\text { angle } \\
(\varphi)\end{array}$ & $\mathrm{H}=10 \mathrm{~m}$ & $\mathrm{H}=25 \mathrm{~m}$ & $\begin{array}{c}\mathrm{H}=100 \\
\mathrm{~m}\end{array}$ & $\begin{array}{c}\mathrm{H}=250 \\
\mathrm{~m}\end{array}$ & $\mathrm{H}=\infty$ \\
\cline { 2 - 6 } & $7.6 \%$ & $1 \%$ & $-3.6 \%$ & $-1.8 \%$ & $6.7 \%$ \\
35 & $11.5 \%$ & $6.12 \%$ & $1.9 \%$ & $1.6 \%$ & $5.6 \%$ \\
15 & $6 \%$ & $5.8 \%$ & $4.6 \%$ & $3.8 \%$ & $5.27 \%$ \\
10 & $-8.1 \%$ & $0.3 \%$ & $3.6 \%$ & $3.1 \%$ & $8.35 \%$ \\
\hline
\end{tabular}

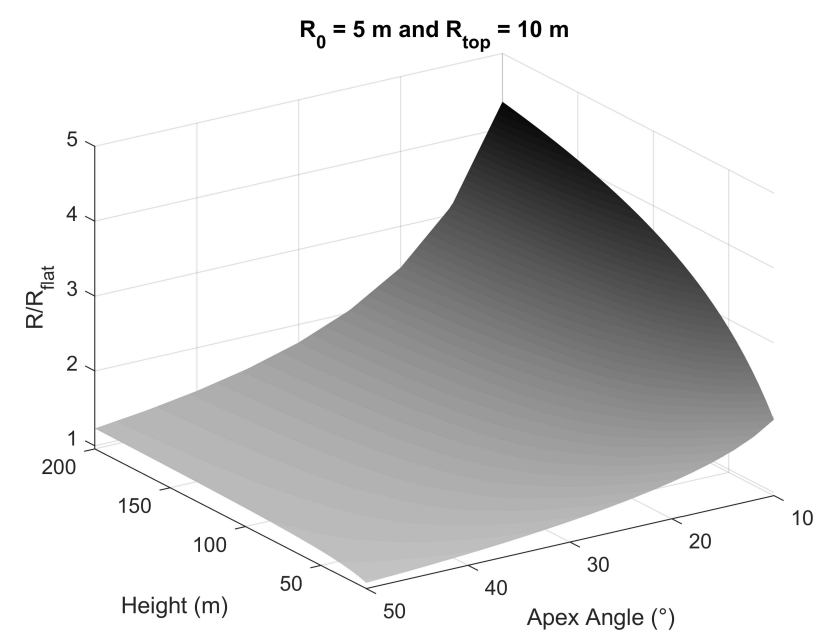

Fig. 2. Increase of the grounding resistance predicted by the derived analytical formula as a function of the height of the truncated cone and its apex angle, for a $5-\mathrm{m}$ radius hemispheric electrode. The top radius of the truncated cone is 10 $\mathrm{m}$.

\section{CONCLUSION}

Recently, closed-form solutions for the grounding resistance have been derived first for a hemispheric electrode on top of a cone, and later for a more realistic case of a truncated cone with a flat region at its top. The height of the cone was considered infinite in those studies.

In this paper, we derived an analytical solution for the grounding resistance of a hemispheric electrode located on a mountaintop represented by a truncated cone, taking into account its finite height. The derived analytical solution was validated using as reference numerical simulations.

The effect of the cone height and apex angle on the resulting grounding resistance was discussed. The relative error incurred in when using the derived approximate analytical expression for the grounding resistance is much smaller than $10 \%$ for the cases studied in this paper with the exception of a single case for which the error was of the order of $10 \%$.

Acknowledgments - This work was supported in part by the Swiss National Science Foundation (Project No. 200020_175594). 


\section{REFERENCES}

[1] A. Smorgonskiy, F. Rachidi, M. Rubinstein, and G. Diendorfer, "On the relation between lightning flash density and terrain elevation," in 2013 International Symposium on Lightning Protection (XII SIPDA), 2013.

[2] D. Poljak, D. Cavka, Properties and Characterization of Modern Materials, Chapter Electromagnetic Compatibility Aspects of Wind Turbine Analysis and Design July 2016.

[3] F. Rachidi, M. Rubinstein, J. Montanyà, J. Bermúdez, R.Sola, G. Solà, and N. Korovkin, "A Review of Current Issues in Lightning Protection of New-Generation Wind-Turbine Blades", IEEE Transactions on Industrial Electronics, Vol. 55, No. 6, June 2008.

[4] F. Rachidi, M. Rubinstein and A. Smorgonskiy, Lightning Protection of Large Wind-Turbine Blades, in Wind Energy Conversion Systems, Springer, p. 227-242, 2012.

[5] "IEC International Standard. Lightning protection. IEC 61400e61424" in Wind turbine generation system, Geneva:International Electrotechnical Commission, vol. 24, 2010.

[6] Motorola R56, Standards and Guidelines for Communication Sites, 2005

[7] D. Cavka, D. Poljak, V. Doric, and R. Goic, "Transient analysis of grounding systems for wind turbines," Renewable Energy, vol. 43, pp. 284-291, Jul. 2012.R.

[8] Alipio and S. Visacro, "Modeling the Frequency Dependence of Electrical Parameters of Soil," IEEE Transactions on Electromagnetic Compatibility, vol. 56, no. 5, pp. 1163-1171, Oct. 201

[9] F. P. Dawalibi, J. Ma, and R. D. Southey, "Behaviour of grounding systems in multilayer soils: a parametric analysis," IEEE Transactions on Power Delivery, vol. 9, no. 1, pp. 334-342, 1994.

[10] A. M. Mousa, "The soil ionization gradient associated with discharge of high currents into concentrated electrodes," IEEE Transactions on Power Delivery, vol. 9, no. 3, pp. 1669-1677, Jul. 1994.

[11] A. Sunjerga, F. Rachidi, M. Rubinstein, and D. Poljak, "Calculation of the Grounding Resistance of Structures Located on Elevated Terrain," IEEE Transactions on Electromagnetic Compatibility, pp. 1-5, 2018.

[12] A. Sunjerga, F. Rachidi, M. Rubinstein, and D. Poljak, "On the Influence of an Elevated Terrain on the Grounding Resistance of a Vertical Rod" presented at 19th International Conference on Environment and Electrical Engineering (EEEIC), 2019.

[13] A. Sunjerga, F. Rachidi, M. Rubinstein, and D. Poljak, "Reduction of the Cone Shaped Terrain Grounding Resistance by Remote Grounding" submitted to 27th International Conference on Software, Telecommunications and Computer Networks (SoftCOM), 2019.

[14] E. D. Sunde, Earth Conduction Effects in Transmission Systems. New York: Van Nostrand, 1949.

[15] L. Grcev and V. Arnautovski-Toseva, "Grounding systems modeling for high frequencies and transients: some fundamental considerations," in 2003 IEEE Bologna Power Tech Conference Proceedings

[16] L. D. Grcev, A. Kuhar, V. Arnautovski-Toseva, and B. Markovski, "Evaluation of High-Frequency Circuit Models for Horizontal and Vertical Grounding Electrodes," IEEE Transactions on Power Delivery, pp. 1-1, 2018.

[17] F. M. Tesche, M. Ianoz, T. Karlsson EMC Analysis Methods and Computational Models, N.J. Wiley, 1997.

[18] Tan, K. B., Lu, H. M., Zhang, Y., \& Zuo, W. C. (2019). Analysis of the Grounding Resistance of a Hemispheric Electrode Located on a Truncated Cone. IEEE Transactions on Electromagnetic Compatibility, 1-3. https://doi.org/10.1109/temc.2019.2940902

[19] COMSOL Multiphysics ${ }^{\circledR}$ v.5.3. www.comsol.com. COMSOL AB, Stockholm, Sweden. 